Original Research Article

\title{
Evaluation of effectiveness of rupatadine and olopatadine in patients of allergic rhinitis at Indore: a randomized control trial
}

\author{
Jay Shah, Poonam Patel*
}

Department of Pharmacology, Index Medical College Hospital and Research Centre, Indore, Madhya Pradesh, India

Received: 05 May 2017

Revised: 19 May 2017

Accepted: 31 May 2017

*Correspondence to:

Dr. Poonam Patel,

Email: researchguide86@ gmail.com

Copyright: (C) the author(s), publisher and licensee Medip Academy. This is an openaccess article distributed under the terms of the Creative Commons Attribution NonCommercial License, which permits unrestricted noncommercial use, distribution, and reproduction in any medium, provided the original work is properly cited.

\begin{abstract}
Background: Allergic rhinitis (AR) is one of the most prevalent atopic disorders that affect productivity and quality of life. Although individually olopatadine and rupatadine are efficacious in AR, data on the head to head study of these drugs in Indian population are scanty, so present study was undertaken with an aim to compare the efficacy, safety, and cost effectiveness of rupatadine and olopatadine in patients' of allergic rhinitis.

Methods: A prospective, randomized, double blind, parallel group, comparative 2 weeks clinical study conducted in eighty patients of AR in the Department of Pharmacology, Index Medical College Hospital and Research Centre, Indore. Following inclusion and exclusion criteria, 67 patients were recruited and randomized to two treatment groups and received the respective drugs for 2 weeks. At follow-up, parameters assessed were total nasal symptom score (TNSS), change in total and differential count of eosinophil.

Results: In olopatadine group, there was a significantly higher reduction in TNSS $(\mathrm{P}<0.05)$ than that of rupatadine. Both the drugs significantly reduced the absolute eosinophil count, but olopatadine $(\mathrm{P}<0.001)$ was found to be superior. The incidence of adverse effects was found to be less in olopatadine group when compared with rupatadine group.

Conclusions: Both rupatadine and olopatadine provide effective relief of the symptoms of AR. However, clinical benefit occurs significantly more with olopatadine. However, rupatadine was more cost-effective than olopatadine in treating the patients of AR in term of effectiveness.
\end{abstract}

Keywords: Allergic rhinitis, Efficacy, Indore, Randomized

\section{INTRODUCTION}

Allergic rhinitis (AR) is one of the most prevalent atopic disorders that affect productivity and quality of life. AR is characterized by sneezing, itching, rhinorrhea, nasal congestion, nasal hypersensitivity, and non-nasal symptoms such as itching and watery eyes or itching ears and palate, and signs of invasion of nasal mucosa by inflammatory cells. ${ }^{1}$ The prevalence of AR varies from population to population, but on an average, it can affect $25 \%-35 \%$ of people. AR is associated with sleep disturbances that result in impaired work productivity and interference with cognitive and emotional functioning. ${ }^{2}$
Today's therapy is based on three measures, mainly avoidance or elimination of the causative agent, symptomatic pharmacotherapy, and specific immunotherapy. The new patient should be managed differently according to the duration and severity of the clinical picture, patient's personal preferences, accessibility and the affordability of medications, and the success and effectiveness of a therapeutic option applied in the patient's particular case. The current therapeutic modalities for the management of AR include: H1 receptor antagonists, decongestants, mast cell stabilizers, leukotriene receptor antagonists, corticosteroids, and 
anticholinergic agents in oral or topical nasal formulations. ${ }^{3}$

Two new second-generation H1-receptor antagonists, olopatadine and rupatadine, are known as dual blockers since both these drugs block the action of not only the histamine but also of other inflammatory mediators such as platelet-activating factor (PAF), LTs, and chemokines. Olopatadine is a newly approved drug for the treatment of AR. It is a selective histamine H1-receptor antagonist, in addition possessing inhibitory effects on PAF and on the release of inflammatory lipid mediators such as LT and thromboxane (TX) from human polymorphonuclear leukocytes and eosinophils. ${ }^{2}$ Olopatadine was shown to be highly useful for the treatment of AR, chronic urticaria, and conjunctivitis in double-blind clinical trials. $^{4,5}$ Rupatadine is a selective and long-acting oral histamine H1-receptor antagonist that has also been shown to have PAF antagonist activity. ${ }^{6}$ It is indicated for use in AR and chronic idiopathic urticaria in patients aged 12 years or more. ${ }^{7}$

Although individually olopatadine and rupatadine are efficacious in AR, data on the head to head study of these drugs in Indian population are scanty. In spite of extensive literature search, we could not find any study comparing the cost effectiveness of these drugs. Therefore, we aimed to compare the efficacy and safety of olopatadine with that of rupatadine and to determine the cost effectiveness so that better option will be offered for the treatment of AR.

\section{METHODS}

A prospective, randomized, double-blind, parallel group, comparative 2 weeks clinical study conducted in eighty patients of AR in the Department of Pharmacology, Index Medical College Hospital and Research Centre, Indore. Ethical clearance was obtained from the institutional ethics board and written informed consent was taken from all the participants. Patients were divided into two groups with forty patients in each group to receive either olopatadine $10 \mathrm{mg}$ or rupatadine $10 \mathrm{mg}$ once daily orally for 2 weeks. $^{2}$ Study was conducted during January 2014 to December 2014. After the written informed consent was obtained from each patient, patients were enrolled in the study.

\section{Inclusion criteria}

- Patients between 18 and 65 years of either gender with history of having intermittent or persistent mild, moderate, to severe AR.

- Patients with TNNS of $\geq 8$ not treated with antihistamines in the last 3 days.

- Patients who could understand and were able to adhere to the dosing and visit schedules.

\section{Exclusion criteria}

- $\quad$ Patients with H/O asthma requiring chronic use of inhaled or systemic corticosteroids had been unresponsive to antihistamine treatment in the past.

- Patients with history of allergies to study medication or unable to tolerate antihistamines.

- Use of study drug in the last 3 days before baseline.

After clinical diagnosis of AR, baseline investigations were carried out and patients were given drugs for 2 weeks. After 2 weeks, again investigations were repeated, and data were analyzed. In total, $10 \mathrm{ml}$ blood of each patient was withdrawn by taking all aseptic precautions at both 0 and 2 weeks. Diagnosis of AR was made on the basis of patient's chief complaints and history. Those meeting the inclusion criteria were briefed about the study. A patient information sheet was given to all prospective participants.

After initial screening, clinical examination, and laboratory investigations, patients were randomly allocated to receive either olopatadine (Group A) or rupatadine (Group B). Block randomization procedure was used for random allocation of study drugs. Block size of four patients was taken and divided in equal proportions in Groups A and B to ensure uniform allocation ratio of 1:1. All patients received one capsule filled with either olopatadine $10 \mathrm{mg}$ or rupatadine $10 \mathrm{mg}$ once a day at 10 p.m. Clinical findings and laboratory investigations were recorded. Drugs were purchased by the principal investigator and distributed to the patients free of cost.

Double blinding was done by inserting olopatadine or rupatadine tablet in a nontransparent capsule of the same size, shape, and color. These capsules were given to a third person not directly involved in this study for coding. Codes were maintained by this person and revealed at the end of the study after the data analysis. Clinical assessment of patients was done by the principal investigator and the consultants. Patients were assessed for total nasal symptom score (TNSS) at each visit by the principal investigators. Symptom severity was determined by the TNSS which consisted of sneezing, rhinorrhea, itching, and nasal congestion scored on a severity scale from 0 to $3(0=$ none, $1=$ mild, $2=$ moderate, and $3=$ severe), such that the maximum possible TNSS was $12 .^{2,8}$

Total and differential leukocyte counts, renal and liver function tests and electrocardiogram were performed at first visit $\left(0\right.$ week) and last visit $\left(2^{\text {nd }}\right.$ week). Concomitant medication restriction Participants were advised to avoid concomitant medication with other antiallergic therapy or with drugs depressing the central nervous system, such as hypnotics or sedatives, in the last $72 \mathrm{~h}$, topical corticosteroids in the last 7 days, oral corticosteroids in the last 8 weeks, or parenteral corticosteroids in the last 3 months. Assessment of cost effectiveness for cost 
effectiveness analysis, cost effectiveness ratio of both treatment groups was calculated based on formula Cost effectiveness $=$ cost/outcome. ${ }^{9}$

\section{Statistical analysis}

The data was coded and entered into Microsoft Excel spreadsheet. Analysis was done using SPSS version 15 (SPSS Inc. Chicago, IL, USA) Windows software program. Descriptive statistics included computation of percentages. For all tests, confidence level and level of significance were set at $95 \%$ and $5 \%$ respectively. Statistical tests used were nonparametric Wilcoxon test, Mann-Whitney Rank Sum test, Fisher's exact test, and parametric paired t-test and unpaired t-test.

\section{RESULTS}

In total, eighty patients were recruited. Sixty-seven patients completed the study. Thirteen patients lost to follow-up by the end of the study, 6 in olopatadine group and 7 in rupatadine group. Patients were randomly assigned to the treatment with olopatadine (Group A) or rupatadine (Group B).

The percentage of male patients was relatively more than female patients, $61.76 \%$ in olopatadine group and $55.88 \%$ in rupatadine group. Both groups were comparable, and there were no statistically significant differences between two groups at baseline (Table 1).

Table 1: Comparison of effects of drugs after 2 weeks of treatment taking into consideration the change from baseline values.

\begin{tabular}{|llll|}
\hline Characteristic & $\begin{array}{l}\text { Olopatadine } \\
\text { group } \\
(\mathbf{n = 3 4 )} \\
\text { Mean (SD) }\end{array}$ & $\begin{array}{l}\text { Rupatadine } \\
\text { group } \\
\text { (n=33) } \\
\text { Mean (SD) }\end{array}$ & $\begin{array}{l}\text { P } \\
\text { value }\end{array}$ \\
\hline Age (years) & $37.21(8.34)$ & $36.23(9.54)$ & 0.43 \\
\hline TLC & $8110(964.2)$ & $8195(1102)$ & 0.54 \\
\hline Neutrophils(\%) & $63.31(4.22)$ & $61.99(5.43)$ & 0.50 \\
\hline $\begin{array}{l}\text { Lymphocytes } \\
\text { (\%) }\end{array}$ & $29.56(5.70)$ & $29.47(5.70)$ & 0.12 \\
\hline Eosinophils(\%) & $6.82(1.50)$ & $6.62(1.52)$ & 0.67 \\
\hline Monocytes(\%) & $0.89(0.97)$ & $0.72(0.90)$ & 0.89 \\
\hline Basophils(\%) & $0.58(0.85)$ & $0.57(0.70)$ & 0.76 \\
\hline $\begin{array}{l}\text { Serum } \\
\text { bilirubin(mg\%) }\end{array}$ & $0.64(0.20)$ & $0.62(0.22)$ & 0.99 \\
\hline $\begin{array}{l}\text { Blood urea } \\
\text { (mg\%) }\end{array}$ & $20.71(3.73)$ & $19.90(4.95)$ & 0.23 \\
\hline $\begin{array}{l}\text { Serum } \\
\text { creatinine } \\
\text { (mg\%) }\end{array}$ & $0.76(0.22)$ & $0.85(0.23)$ & 0.11 \\
\hline
\end{tabular}

Statistical Significance at $\mathrm{p} \leq 0.05$

There was no statistically significant difference between olopatadine and rupatadine groups in TNSS at baseline. However, TNSS in olopatadine and rupatadine groups at baseline and $2^{\text {nd }}$ week revealed statistically significant difference after 2 weeks of treatment with olopatadine and rupatadine.

To test whether rupatadine or olopatadine is better at reducing the TNSS, we compared the effects of drugs after 2 weeks of treatment taking into consideration the change from baseline values. Between-group comparison showed that reduction in TNNS at 2 weeks was more ( $\mathrm{P}$ $<0.05)$ with olopatadine as compared to rupatadine There was a significant decrease in neutrophil count, eosinophil count, and increase in lymphocyte count after 2 weeks of treatment in olopatadine group. While in rupatadine group, only eosinophil count decreased significantly after 2 weeks of treatment. Reduction in eosinophil count was more in olopatadine group as compared to rupatadine group at 2 weeks of treatment $(\mathrm{P}<0.05)$. There was no significant change in liver and kidney function after 2 weeks of treatment in both groups as compared to baseline. Sedation was the most common adverse event in both groups. Headache and dryness of mouth were noted in both groups while gastric irritation was noted in olopatadine group. For cost-effectiveness analysis, only direct health cost of drug treatment was taken into consideration. Calculating the total cost of treatment at the end 2nd week gave us the idea of difference of total cost in the treatment which is more in olopatadine group. ${ }^{9}$

The treatment modality having less cost-effectiveness ratio is considered as superior. In our study, the cost effectiveness ratio is less in rupatadine group at the $2^{\text {nd }}$ week stating that the rupatadine is more cost-effective.

\section{DISCUSSION}

It remains a challenge to the physicians to treat the symptoms of AR and ensure a good quality of life to the patients. An increase in the understanding of the pathomechanisms of $\mathrm{AR}$ in the last few decades has revealed the potential of the new generation antihistamines with dual blocking property in the treatment of AR. Rupatadine and olopatadine are known to be effective in AR in several clinical trials, but this study was performed to compare their efficacy and safety and thus to choose the better agent.

The TNSS is a widely accepted and reliable tool to assess the efficacy of a drug in the treatment of AR, and a decrease in the score suggests that there is an overall clinical improvement in the condition. We observed a significant difference in TNSS after completion of the treatment in olopatadine as well as rupatadine groups. Rescue medication was not required in any of the subjects. In the present study, a significant and progressive decrease in the TNSS was observed at the end of 2 weeks of treatment with olopatadine and rupatadine. In a study conducted to test the efficacy of rupatadine in treating patients with $A R$, rupatadine was found to be effective in reducing nasal symptoms, improving signs secondary to mucosal inflammation with 
sustained and even improving results after 2 weeks of treatment. ${ }^{6}$ Another comparative dose ranging trial of rupatadine showed improvement in nasal and ocular symptoms of AR. ${ }^{10}$ Our study supports this finding of a significant reduction in symptom score and improvement in nasal symptoms.

In a randomized, double-blind, placebo controlled study, oral olopatadine significantly suppressed sneezing ( $\mathrm{P}$ $<0.001)$, rhinorrhea $(\mathrm{P}<0.001)$, and nasal congestion ( $\mathrm{P}$ $<0.05){ }^{4}$ In another comparative open labeled study, olopatadine and rupatadine have decreased the TNSS, but olopatadine was found to be superior to rupatadine in reducing the TNSS. ${ }^{2}$ Our study supports this finding of significant reduction in TNSS with rupatadine and olopatadine both. Change in TNSS was significant and more pronounced in olopatadine than in rupatadine group.

Olopatadine and rupatadine are known as dual blockers since both these drugs block the action of not only the histamine but also of other inflammatory mediators such as PAF, LTs, and chemokines. The probable superiority of olopatadine over rupatadine may be attributed to the following findings. Olopatadine can reduce the amount of cell associated PAF by $52.8 \%$, which is more than rupatadine. ${ }^{11}$ PAF known to increase vascular permeability and is increasingly recognized as an important mediator in inflammation. It also suppresses LTs and TxA2 release, and PAF formation by reducing arachidonic acid release from membrane phospholipids, probably through interference with phospholipase A2.12 Olopatadine has been shown to suppress the activity of S100A12, which is a member of the S100 family of calcium-binding proteins, and exerts multiple pro inflammatory activities including chemotaxis for monocytes and neutrophils. ${ }^{13}$ All these actions inhibit the inflammatory mediators which are the main contributors for vasodilation, vascular leakage, and eosinophil chemotaxis, thus decreasing the symptoms of AR by reducing number of sneezing, itching, rhinorrhea, and nasal blockade more significantly as compared to rupatadine.

Allergic conditions are usually associated with the changes in the percentage of eosinophil and its absolute count and probably that's why the effects of the drugs have not been directly reflected on total leukocyte count and neutrophil count. The increase in eosinophil count is the hallmark of the late phase of AR. The scrupulous control of this parameter is an important therapeutic decrease in differential eosinophil count in both groups. The comparative change in the differential count of eosinophil in olopatadine group was found to be significantly more as compared to the rupatadine group. This observation supports the previous study of analysis of rupatadine and olopatadine. ${ }^{6}$

There was no significant difference in differential monocyte count and differential basophil count in both groups. In our study, we did not find any significant change in biochemical parameters. This observation supports the previous study on safety analysis of rupatadine and olopatadine. ${ }^{2}$ Most frequently occurring adverse event with rupatadine was sedation, which supports the previous studies conducted on rupatadine for its safety profile. Other adverse events noted were headache, gastric irritation, and dryness of mouth. ${ }^{14}$ Similarly, with olopatadine, the most commonly occurring adverse event was sedation, which supports the finding of the previous study. Our study did not reveal any change in electrocardiogram (ECG), supporting the previous studies which also showed no change in ECG findings. ${ }^{15}$

To compare the cost effectiveness of two drugs, only the direct health cost of the drug treatment was taken into consideration. When we compared the cost effectiveness ratio of the treatment, i.e., rupatadine and olopatadine, we found that cost effectiveness ratio was less in rupatadine. For pharmacoeconomic analysis, treatment modality having less cost effectiveness ratio is considered as superior. Thus, it suggests that rupatadine is more cost-effective than olopatadine for the treatment of AR. However, after an in-depth search, we could not find any study related to our finding. This information can help physicians in selecting a drug for treating the patients of AR. Patients, who can afford costly treatment, can be prescribed more effective olopatadine, while for patients who cannot afford; it can be prescribed rupatadine as it is more cost-effective.

Although the present study was double blind with small sample size and of short duration, the results of the study cannot be ignored. However, studies with larger sample size and longer follow up periods, along with measurements of absolute eosinophils counts, may yield more meaningful data to compare rupatadine and olopatadine.

\section{CONCLUSION}

Both rupatadine and olopatadine provide effective relief of the symptoms of AR. However, clinical benefit occurs significantly more with olopatadine. It may be due to additional PAF antagonistic property and anti-inflammatory effects of olopatadine that act directly on the $\mathrm{H} 1$ receptor. However, rupatadine was more cost-effective than olopatadine in treating the patients of $\mathrm{AR}$ in term of effectiveness.

Funding: No funding sources

Conflict of interest: None declared

Ethical approval: The study was approved by the Institutional Ethics Committee

\section{REFERENCES}

1. Maiti R, Rahman J, Jaida J, Allala U, Palani A. Rupatadine and levocetirizine for seasonal allergic 
rhinitis: A comparative study of efficacy and safety. Arch Otolaryngol Head Neck Surg. 2010;136:796-800.

2. Maiti R, Jaida J, Rahman J, Gaddam R, Palani A. Olopatadine hydrochloride and rupatadine fumarate in seasonal allergic rhinitis: A comparative study of efficacy and safety. J Pharmacol Pharmacother. 2011;2:270-6.

3. Dykewicz MS, Fineman S, Skoner DP, Nicklas R, Lee $\mathrm{R}$, Blessing-Moore $\mathrm{J}$, et al. Diagnosis and management of rhinitis: Complete guidelines of the Joint Task Force on Practice Parameters in Allergy, Asthma and Immunology. American Academy of Allergy, Asthma, and Immunology. Ann Allergy Asthma Immunol. 1998;81(5 Pt 2):478-518.

4. Yamamoto H, Yamada T, Kubo S, Osawa Y, Kimura $\mathrm{Y}$, Oh $\mathrm{M}$, et al. Efficacy of oral olopatadine hydrochloride for the treatment of seasonal allergic rhinitis: A randomized, double-blind, placebo-controlled study. Allergy Asthma Proc. 2010;31:296-303.

5. Enomoto T, Lu HQ, Yin M, Sakoda T, Dake Y, Enomoto K, et al. Evaluation of the efficacy and safety of olopatadine and fexofenadine compared with placebo in Japanese cedar pollinosis using an environmental exposure unit. J Investig Allergol Clin Immunol. 2009;19:299-305.

6. Mion Ode G, Campos RA, Antila M, Rapoport PB, Rosario N, de Mello Junior JF, et al. Futura study. Evaluation of efficacy and safety of rupatadine fumarate in the treatment of persistent allergic rhinitis. Braz J Otorhinolaryngol. 2009;75:673-9.

7. Valero A, Izquierdo I, Giralt J, Bartra J, del Cuvillo A, Mullol J. Rupatadine improves nasal symptoms, quality of life (ESPRINT-15) and severity in a subanalysis of a cohort of Spanish allergic rhinitis patients. J Investig Allergol Clin Immunol. 2011;21:229-35.

8. Kubavat AH, Pawar P, Mittal R, Sinha V, Shah UB, Ojha $\mathrm{T}$, et al. An open label, active controlled, multicentric clinical trial to assess the efficacy and safety of fluticasone furoate nasal spray in adult
Indian patients suffering from allergic rhinitis. J Assoc Physicians India. 2011;59:424-8.

9. Cohen DJ, Reynolds MR. Interpreting the results of cost-effectiveness studies. J Am Coll Cardiol. 2008;52:2119-26.

10. Katiyar S, Prakash S. Pharmacological profile, efficacy and safety of rupatadine in allergic rhinitis. Prim Care Respir J. 2009;18:57-68.

11. Ohmori K, Hayashi K, Kaise T, Ohshima E, Kobayashi S, Yamazaki T, et al. Pharmacological, pharmacokinetic and clinical properties of olopatadine hydrochloride, a new antiallergic drug. Jpn J Pharmacol. 2002;88:379-97.

12. Miyake K, Ohmori K, Ishii A, Karasawa A. Short communication inhibitory effect of olopatadine hydrochloride (KW-4679), a novel antiallergic drug, on peptide leukotriene release from human eosinophils. Allergol Int. 2001;50:113-6.

13. Kishimoto K, Kaneko S, Ohmori K, Tamura T, Hasegawa K. Olopatadine suppresses the migration of THP-1 monocytes induced by S100A12 protein. Mediators Inflamm. 2006:42726.

14. Dakhale GN, Wankhede SS, Mahatme MS, Hiware SK, Mishra DB, Dudhgaonkar SS. Comparison of efficacy, safety and cost-effectiveness of rupatadine and olopatadine in patients of chronic spontaneous urticaria: A Randomized, Double-blind, Comparative, Parallel Group Trial. Indian J Dermatol. 2016;61:63-9.

15. Donado E, Izquierdo I, Pérez I, García O, Antonijoan RM, Gich I, et al. No cardiac effects of therapeutic and supratherapeutic doses of rupatadine: Results from a 'thorough QT/QTc study' performed according to $\mathrm{ICH}$ guidelines. Br J Clin Pharmacol. 2010;69:401-10

Cite this article as: Shah J, Patel P. Evaluation of effectiveness of rupatadine and olopatadine in patients of allergic rhinitis at Indore: a randomized control trial. Int J Basic Clin Pharmacol 2017;6:1753-7. 